\title{
Influence of Density functionals and basis sets on One-Bond Carbon-Carbon NMR Spin-Spin Coupling constants.
}

\author{
R. Suardíaz ${ }^{a}$, C. Pérez ${ }^{a}$, R. Crespo-Otero ${ }^{a}$, \\ José M. García de la Vega ${ }^{b}$, and Jesús San Fabián ${ }^{b}$ \\ ${ }^{a}$ Dpto. de Química Física, Facultad de Química, Universidad de la Habana. \\ La Habana 10400, Cuba. \\ ${ }^{b}$ Dpto. de Química Física Aplicada, Facultad de Ciencias, \\ Universidad Autónoma de Madrid, 28049 Madrid, Spain.
}

$2007 / 11 / 29$ 
Table 1: One-bond $\mathrm{C}-\mathrm{C}$ coupling constants (in $\mathrm{Hz}$ ). Experimental and values calculated using PBE functional and 6-311G**, TZVP, EPR-III and aug-cc-pVTZ-J basis sets.

\begin{tabular}{|c|c|c|c|c|c|c|c|}
\hline \multirow[t]{2}{*}{ Molec. } & \multirow[t]{2}{*}{ Coupling } & \multirow[t]{2}{*}{ Type } & \multirow[t]{2}{*}{${ }^{1} J_{C C}^{e x p}$} & \multicolumn{4}{|c|}{${ }^{1} J_{C C}^{c a l}$ PBE functional } \\
\hline & & & & $6-311 G^{* *}$ & TZVP & EPR-III & aug-cc-pVTZ-J \\
\hline 1 & & $s p^{3}-s p^{3}$ & $34.6^{\mathrm{a}}$ & 29.48 & 27.43 & 26.46 & 27.37 \\
\hline 2 & & $s p^{3}-s p^{3}$ & $38.2^{\mathrm{a}}$ & 33.07 & 30.56 & 29.37 & 30.50 \\
\hline 3 & 1,2 & $s p^{3}-s p^{3}$ & $33.0^{\mathrm{a}}$ & 28.27 & 26.58 & 25.70 & 26.59 \\
\hline 4 & & $s p^{3}-s p^{3}$ & $12.4^{\mathrm{b}}$ & 10.54 & 7.67 & 7.65 & 8.08 \\
\hline 5 & & $s p^{3}-s p^{3}$ & $28.0^{\mathrm{c}}$ & 25.09 & 22.00 & 21.16 & 22.13 \\
\hline 6 & & $s p^{3}-s p^{3}$ & $21.0^{\mathrm{c}}$ & 19.14 & 16.09 & 15.59 & 16.33 \\
\hline 7 & 1,2 & $s p^{3}-s p^{3}$ & $10.9^{\mathrm{a}}$ & 8.62 & 6.47 & 6.64 & 7.00 \\
\hline 8 & & $s p^{3}-s p^{3}$ & $28.4^{\mathrm{b}}$ & 23.31 & 20.86 & 20.71 & 21.39 \\
\hline 9 & & $s p^{3}-s p^{3}$ & $29.54^{\mathrm{a}}$ & 23.59 & 21.24 & 21.28 & 21.97 \\
\hline 10 & 1,2 & $s p^{3}-s p^{3}$ & $21.0^{\mathrm{a}}$ & 17.74 & 16.41 & 15.83 & 16.59 \\
\hline 10 & 1,3 & $s p^{3}-s p^{3}$ & $-17.49^{\mathrm{a}}$ & -14.84 & -17.37 & -15.61 & -15.96 \\
\hline 11 & 1,2 & $s p^{3}-s p^{3}$ & $22^{\mathrm{a}}$ & 18.69 & 17.52 & 16.92 & 17.68 \\
\hline 12 & 1,2 & $s p^{3}-s p^{3}$ & $36.7^{\mathrm{a}}$ & 30.14 & 29.13 & 28.33 & 29.32 \\
\hline 12 & 2,3 & $s p^{3}-s p^{3}$ & $16.0^{\mathrm{a}}$ & 12.67 & 11.25 & 11.03 & 11.59 \\
\hline 13 & 1,2 & $s p^{3}-s p^{3}$ & $20.2^{\mathrm{d}}$ & 18.27 & 15.46 & 14.90 & 15.66 \\
\hline 14 & 1,2 & $s p^{3}-s p^{3}$ & $9.9^{\mathrm{d}}$ & 5.58 & 4.02 & 4.49 & 4.91 \\
\hline 15 & & $s p^{3}-s p^{3}$ & $32.6^{\mathrm{b}}$ & 27.64 & 26.19 & 25.32 & 26.19 \\
\hline 16 & & $s p^{3}-s p^{3}$ & $29.4^{\mathrm{e}}$ & 20.96 & 20.51 & 20.86 & 21.46 \\
\hline 17 & 1,2 & $s p^{3}-s p^{3}$ & $25.3^{\mathrm{f}}$ & 17.51 & 17.38 & 17.39 & 17.98 \\
\hline 18 & 1,2 & $s p^{3}-s p^{2}$ & $41.9^{\mathrm{a}}$ & 35.64 & 34.36 & 33.71 & 34.88 \\
\hline 19 & 1,2 & $s p^{3}-s p^{2}$ & $44.9^{\mathrm{a}}$ & 39.43 & 38.00 & 36.10 & 37.45 \\
\hline 20 & 1,3 & $s p^{3}-s p^{2}$ & $44.19^{\mathrm{a}}$ & 39.47 & 38.32 & 36.28 & 37.56 \\
\hline 21 & & $s p^{3}-s p^{2}$ & $40.51^{\mathrm{a}}$ & 35.25 & 32.71 & 31.18 & 32.37 \\
\hline 22 & & $s p^{3}-s p^{2}$ & $48.48^{\mathrm{a}}$ & 47.89 & 46.23 & 43.49 & 45.13 \\
\hline 23 & 1,2 & $s p^{3}-s p^{2}$ & $49.3^{\mathrm{a}}$ & 47.79 & 46.54 & 43.78 & 45.46 \\
\hline 23 & 1,3 & $s p^{3}-s p^{2}$ & $41.4^{\mathrm{a}}$ & 35.88 & 33.66 & 32.07 & 33.30 \\
\hline 24 & 1,2 & $s p^{3}-s p^{2}$ & $45.91^{\mathrm{a}}$ & 41.41 & 40.43 & 38.26 & 39.59 \\
\hline 25 & & $s p^{3}-s p^{2}$ & $40.44^{\mathrm{a}}$ & 34.98 & 32.97 & 31.46 & 32.75 \\
\hline 26 & & $s p^{3}-s p^{2}$ & $39.4^{\mathrm{a}}$ & 34.62 & 31.77 & 30.25 & 31.50 \\
\hline 27 & & $s p^{3}-s p^{2}$ & $56.7^{\mathrm{a}}$ & 54.20 & 52.47 & 49.67 & 51.51 \\
\hline 3 & 2,3 & $s p^{3}-s p$ & $55.24^{\mathrm{a}}$ & 56.60 & 52.68 & 49.56 & 51.35 \\
\hline 7 & 2,3 & $s p^{3}-s p$ & $77.9^{\mathrm{a}}$ & 79.81 & 77.54 & 72.50 & 75.05 \\
\hline 28 & & $s p^{3}-s p$ & $56.48^{\mathrm{a}}$ & 58.28 & 54.61 & 51.33 & 53.17 \\
\hline 29 & 1,2 & $s p^{3}-s p$ & $54.8^{\mathrm{a}}$ & 55.41 & 51.79 & 48.73 & 50.49 \\
\hline 30 & 1,2 & $s p^{3}-s p$ & $52.0^{\mathrm{a}}$ & 54.64 & 51.53 & 48.49 & 50.23 \\
\hline 31 & 1,2 & $s p^{3}-s p$ & $67.4^{\mathrm{a}}$ & 67.81 & 65.39 & 61.45 & 63.59 \\
\hline 32 & & $s p^{2}-s p^{2}$ & $67.6^{\mathrm{a}}$ & 72.02 & 67.35 & 62.68 & 65.64 \\
\hline 18 & 2,3 & $s p^{2}-s p^{2}$ & $70.0^{\mathrm{a}}$ & 71.93 & 68.51 & 65.18 & 68.01 \\
\hline 19 & 2,3 & $s p^{2}-s p^{2}$ & $73.8^{\mathrm{a}}$ & 77.16 & 73.56 & 68.48 & 71.61 \\
\hline 20 & 1,2 & $s p^{2}-s p^{2}$ & $57.3^{\mathrm{a}}$ & 57.72 & 54.87 & 50.81 & 53.16 \\
\hline 33 & 1,2 & $s p^{2}-s p^{2}$ & $70.6^{\mathrm{a}}$ & 74.77 & 71.00 & 66.15 & 69.20 \\
\hline
\end{tabular}


Deviations form the experimental ${ }^{1} J_{C C}^{e x p}$ of the scaled coupling constants, continued...

\begin{tabular}{|c|c|c|c|c|c|c|c|}
\hline \multirow[t]{2}{*}{ Molec. } & \multirow[t]{2}{*}{ Coupling } & \multirow[t]{2}{*}{ Type } & \multirow[t]{2}{*}{${ }^{1} J_{C C}^{e x p}$} & \multicolumn{4}{|c|}{${ }^{1} J_{C C}^{c a l}$ PBE functional } \\
\hline & & & & $6-311 G^{* *}$ & TZVP & EPR-III & aug-cc-pVTZ-J \\
\hline 34 & 1,2 & $s p^{2}-s p^{2}$ & $69.1^{\mathrm{a}}$ & 72.26 & 68.70 & 64.40 & 67.31 \\
\hline 35 & 1,2 & $s p^{2}-s p^{2}$ & $65.6^{\mathrm{a}}$ & 69.58 & 66.62 & 62.07 & 64.82 \\
\hline 36 & 1,2 & $s p^{2}-s p^{2}$ & $48.54^{\mathrm{a}}$ & 48.17 & 45.20 & 42.56 & 44.54 \\
\hline 36 & 2,3 & $s p^{2}-s p^{2}$ & $67.4^{\mathrm{a}}$ & 71.84 & 68.13 & 63.81 & 66.71 \\
\hline 37 & 1,2 & $s p^{2}-s p^{2}$ & $58.29^{\mathrm{a}}$ & 50.68 & 47.74 & 44.78 & 46.89 \\
\hline 38 & 1,2 & $s p^{2}-s p^{2}$ & $70.92^{\mathrm{a}}$ & 70.86 & 68.02 & 63.57 & 66.40 \\
\hline 38 & 2,3 & $s p^{2}-s p^{2}$ & $56.63^{\mathrm{a}}$ & 57.74 & 54.76 & 50.82 & 53.16 \\
\hline 38 & 3,4 & $s p^{2}-s p^{2}$ & $56.30^{\mathrm{a}}$ & 57.17 & 54.06 & 50.18 & 52.52 \\
\hline 39 & 1,2 & $s p^{2}-s p^{2}$ & $61.3^{\mathrm{a}}$ & 62.74 & 59.84 & 55.74 & 58.22 \\
\hline 39 & 2,3 & $s p^{2}-s p^{2}$ & $58.1^{\mathrm{a}}$ & 61.02 & 58.16 & 53.82 & 56.25 \\
\hline 39 & 3,4 & $s p^{2}-s p^{2}$ & $56.2^{\mathrm{a}}$ & 57.32 & 54.30 & 50.30 & 52.62 \\
\hline 40 & 1,2 & $s p^{2}-s p^{2}$ & $60.05^{\mathrm{a}}$ & 59.79 & 57.20 & 53.04 & 55.46 \\
\hline 40 & 2,3 & $s p^{2}-s p^{2}$ & $56.47^{\mathrm{a}}$ & 57.37 & 54.29 & 50.32 & 52.66 \\
\hline 40 & 3,4 & $s p^{2}-s p^{2}$ & $55.15^{\mathrm{a}}$ & 55.96 & 52.85 & 48.99 & 51.29 \\
\hline 41 & 1,2 & $s p^{2}-s p^{2}$ & $52.78^{\mathrm{a}}$ & 52.01 & 48.86 & 45.97 & 48.22 \\
\hline 42 & 1,2 & $s p^{2}-s p^{2}$ & $50.39^{\mathrm{a}}$ & 49.85 & 46.23 & 43.68 & 45.82 \\
\hline 43 & 1,2 & $s p^{2}-s p^{2}$ & $57.68^{\mathrm{a}}$ & 59.39 & 56.18 & 52.15 & 54.57 \\
\hline 43 & 1,6 & $s p^{2}-s p^{2}$ & $59.4^{\mathrm{a}}$ & 60.59 & 58.34 & 53.97 & 56.34 \\
\hline 43 & 2,3 & $s p^{2}-s p^{2}$ & $54.71^{\mathrm{a}}$ & 55.19 & 52.18 & 48.47 & 50.73 \\
\hline 43 & 3,4 & $s p^{2}-s p^{2}$ & $57.75^{\mathrm{a}}$ & 59.31 & 56.28 & 52.27 & 54.70 \\
\hline 43 & 4,5 & $s p^{2}-s p^{2}$ & $70.13^{\mathrm{a}}$ & 70.32 & 68.27 & 63.41 & 66.21 \\
\hline 43 & 6,7 & $s p^{2}-s p^{2}$ & $52.2^{\mathrm{a}}$ & 51.71 & 49.56 & 46.14 & 48.06 \\
\hline 44 & 1,2 & $s p^{2}-s p^{2}$ & $57.1^{\mathrm{g}}$ & 63.95 & 58.72 & 55.94 & 58.59 \\
\hline 45 & 1,2 & $s p^{2}-s p^{2}$ & $56.2^{\mathrm{h}}$ & 56.49 & 53.36 & 49.43 & 51.72 \\
\hline 40 & 1,5 & $s p^{2}-s p$ & $80.40^{\mathrm{a}}$ & 81.73 & 79.88 & 74.06 & 76.63 \\
\hline 46 & 1,2 & $s p^{2}-s p$ & $91.1^{\mathrm{a}}$ & 92.73 & 91.99 & 85.57 & 88.63 \\
\hline 47 & & $s p-s p$ & $170.6^{\mathrm{a}}$ & 202.77 & 195.57 & 185.65 & 190.72 \\
\hline 48 & 1,2 & $s p-s p$ & $175.9^{\mathrm{a}}$ & 203.03 & 196.81 & 186.58 & 192.00 \\
\hline 49 & 1,2 & $s p-s p$ & $155.8^{\mathrm{a}}$ & 161.16 & 159.63 & 148.38 & 153.56 \\
\hline 50 & 1,3 & $s p^{3}-s p^{3}$ & $-17.49^{\mathrm{i}}$ & -18.68 & -21.69 & -19.54 & -20.14 \\
\hline 35 & tau. ${ }^{\mathrm{j}}$ & $s p^{2}-s p^{2}$ & $58.29^{\mathrm{a}}$ & 60.33 & 57.28 & 53.47 & 55.91 \\
\hline 51 & 1,2 & $s p^{2}-s p^{2}$ & $59.10^{\mathrm{k}}$ & 61.28 & 56.42 & 53.67 & 56.26 \\
\hline
\end{tabular}

${ }^{\mathrm{a}}$ Wray, V. Prog. NMR Spectrosc. 1979, 13, 177.

${ }^{\mathrm{b}}$ Sauer, S. P. A.; Krivdin, L. B. Magn. Reson. Chem. 2004, 42, 671.

${ }^{\mathrm{c}}$ Krivdin, L. B.; Sauer, S. P. A.; Peralta, J. E.; Contreras, R. H. Magn. Reson. Chem. 2002, $40,187$.

dKrivdin, L. B. Magn. Reson. Chem. 2003, 41, 91.

e Krivdin L. B. Magn. Reson. Chem. 2004, 42, 919.

${ }^{\mathrm{f}}$ Krivdin, L. B. Magn. Reson. Chem. 2003, 41, 417.

gRuden T. A.; Helgaker T.; Jaszuński M. Chem. Phys. 2004, 296, 53.

${ }^{\mathrm{h}}$ Kaski J.; Lantto P.; Vaara J.; Jokisaari J. J. Am. Chem. Soc. 1998, 120, 3993.

${ }^{\mathrm{i}}$ Finkelmeier, H.; Lüttke, W. J. Am. Chem. Soc. 1978, 100, 6261.

${ }^{\mathrm{j}}$ Considering a dynamic tautomerism where the experimental coupling corresponds to the average between ${ }^{1} J_{C_{1}} C_{2}$ and ${ }^{1} J_{C_{1} C_{3}}$ (see Table 5 in paper).

${ }^{k}$ Kamieńska-Trela, K.; Bernatowicz, P.; Lüttke, W.; Machinek, R.; Trætteberg, M. Magn. Reson. Chem. 2002, 40,640 . 
Table 2: One-bond $\mathrm{C}-\mathrm{C}$ coupling constants (in $\mathrm{Hz}$ ). Experimental and values calculated using PW91 functional and 6-311G**, TZVP, EPR-III and aug-cc-pVTZ-J basis set.

\begin{tabular}{|c|c|c|c|c|c|c|c|}
\hline \multirow[t]{2}{*}{ Molec. } & \multirow[t]{2}{*}{ Coupling } & \multirow[t]{2}{*}{ Type } & \multirow[t]{2}{*}{${ }^{1} J_{C C}^{e x p}$} & \multicolumn{4}{|c|}{${ }^{1} J_{C C}^{c a l}$ PW91 functional } \\
\hline & & & & $6-311 \mathrm{G}^{* *}$ & TZVP & EPR-III & aug-cc-pVTZ-J \\
\hline 1 & & $s p^{3}-s p^{3}$ & $34.6^{a}$ & 27.76 & 26.12 & 26.20 & 27.02 \\
\hline 2 & & $s p^{3}-s p^{3}$ & $38.2^{a}$ & 31.29 & 29.20 & 29.13 & 30.13 \\
\hline 3 & 1,2 & $s p^{3}-s p^{3}$ & $33.0^{a}$ & 26.64 & 25.33 & 25.46 & 26.28 \\
\hline 4 & & $s p^{3}-s p^{3}$ & $12.4^{b}$ & 9.33 & 6.73 & 7.39 & 7.76 \\
\hline 5 & & $s p^{3}-s p^{3}$ & $28.0^{c}$ & 23.85 & 21.11 & 21.09 & 22.00 \\
\hline 6 & & $s p^{3}-s p^{3}$ & $21.0^{c}$ & 17.94 & 15.20 & 15.45 & 16.13 \\
\hline 7 & 1,2 & $s p^{3}-s p^{3}$ & $10.9^{a}$ & 7.48 & 5.56 & 6.39 & 6.70 \\
\hline 8 & & $s p^{3}-s p^{3}$ & $28.4^{b}$ & 21.52 & 19.44 & 20.39 & 20.95 \\
\hline 9 & & $s p^{3}-s p^{3}$ & $29.54^{a}$ & 21.80 & 19.85 & 21.00 & 21.59 \\
\hline 10 & 1,2 & $s p^{3}-s p^{3}$ & $21.0^{a}$ & 16.39 & 15.34 & 15.62 & 16.31 \\
\hline 10 & 1,3 & $s p^{3}-s p^{3}$ & $-17.49^{a}$ & -15.39 & -17.89 & -15.95 & -16.33 \\
\hline 11 & 1,2 & $s p^{3}-s p^{3}$ & $22^{a}$ & 17.42 & 16.50 & 16.74 & 17.42 \\
\hline 12 & 1,2 & $s p^{3}-s p^{3}$ & $36.7^{a}$ & 28.36 & 27.70 & 28.07 & 28.95 \\
\hline 12 & 2,3 & $s p^{3}-s p^{3}$ & $16.0^{a}$ & 11.47 & 10.28 & 10.80 & 11.27 \\
\hline 13 & 1,2 & $s p^{3}-s p^{3}$ & $20.2^{d}$ & 16.88 & 14.37 & 14.63 & 15.29 \\
\hline 14 & 1,2 & $s p^{3}-s p^{3}$ & $9.9^{d}$ & 3.98 & 2.63 & 4.02 & 4.34 \\
\hline 15 & & $s p^{3}-s p^{3}$ & $32.6^{b}$ & 26.11 & 25.13 & 25.49 & 26.04 \\
\hline 16 & & $s p^{3}-s p^{3}$ & $29.4^{e}$ & 19.10 & 18.94 & 20.48 & 20.95 \\
\hline 17 & 1,2 & $s p^{3}-s p^{3}$ & $25.3^{f}$ & 16.00 & 16.13 & 17.13 & 17.62 \\
\hline 18 & 1,2 & $s p^{3}-s p^{2}$ & $41.9^{a}$ & 37.40 & 35.68 & 33.88 & 35.16 \\
\hline 19 & 1,2 & $s p^{3}-s p^{2}$ & $44.9^{a}$ & 37.63 & 36.64 & 35.90 & 37.15 \\
\hline 20 & 1,3 & $s p^{3}-s p^{2}$ & $44.19^{a}$ & 37.71 & 36.97 & 36.08 & 37.24 \\
\hline 21 & & $s p^{3}-s p^{2}$ & $40.51^{a}$ & 33.57 & 31.44 & 31.02 & 32.10 \\
\hline 22 & & $s p^{3}-s p^{2}$ & $48.48^{a}$ & 46.00 & 44.82 & 43.27 & 44.83 \\
\hline 23 & 1,2 & $s p^{3}-s p^{2}$ & $49.3^{a}$ & 45.89 & 45.09 & 43.51 & 45.09 \\
\hline 23 & 1,3 & $s p^{3}-s p^{2}$ & $41.4^{a}$ & 34.20 & 32.38 & 31.88 & 33.01 \\
\hline 24 & 1,2 & $s p^{3}-s p^{2}$ & $45.91^{a}$ & 39.64 & 39.06 & 38.06 & 39.28 \\
\hline 25 & & $s p^{3}-s p^{2}$ & $40.44^{a}$ & 33.26 & 31.62 & 31.18 & 32.35 \\
\hline 26 & & $s p^{3}-s p^{2}$ & $39.4^{a}$ & 32.97 & 30.52 & 30.08 & 31.21 \\
\hline 27 & & $s p^{3}-s p^{2}$ & $56.7^{a}$ & 52.40 & 51.14 & 49.57 & 51.32 \\
\hline 3 & 2,3 & $s p^{3}-s p$ & $55.24^{a}$ & 54.60 & 51.18 & 49.43 & 51.10 \\
\hline 7 & 2,3 & $s p^{3}-s p$ & $77.9^{a}$ & 78.01 & 76.27 & 72.69 & 75.11 \\
\hline 28 & & $s p^{3}-s p$ & $56.48^{a}$ & 56.28 & 53.14 & 51.23 & 52.97 \\
\hline 29 & 1,2 & $s p^{3}-s p$ & $54.8^{a}$ & 53.45 & 50.29 & 48.60 & 50.23 \\
\hline 30 & 1,2 & $s p^{3}-s p$ & $52.0^{a}$ & 52.65 & 49.97 & 48.28 & 49.88 \\
\hline 31 & 1,2 & $s p^{3}-s p$ & $67.4^{a}$ & 65.69 & 63.83 & 61.31 & 63.36 \\
\hline
\end{tabular}


Deviations form the experimental ${ }^{1} J_{C C}^{e x p}$ of the scaled coupling constants, continued...

\begin{tabular}{|c|c|c|c|c|c|c|c|}
\hline \multirow[t]{2}{*}{ Molec. } & \multirow[t]{2}{*}{ Coupling } & \multirow[t]{2}{*}{ Type } & \multirow[t]{2}{*}{${ }^{1} J_{C C}^{e x p}$} & \multicolumn{4}{|c|}{${ }^{1} J_{C C}^{c a l}$ PW91 functional } \\
\hline & & & & $6-311 \mathrm{G}^{* *}$ & TZVP & EPR-III & aug-cc-pVTZ-J \\
\hline 32 & & $s p^{2}-s p^{2}$ & $67.6^{a}$ & 69.76 & 65.70 & 62.48 & 65.26 \\
\hline 18 & 2,3 & $s p^{2}-s p^{2}$ & $70.0^{a}$ & 74.12 & 70.12 & 65.28 & 68.30 \\
\hline 19 & 2,3 & $s p^{2}-s p^{2}$ & $73.8^{a}$ & 74.91 & 71.89 & 68.32 & 71.26 \\
\hline 20 & 1,2 & $s p^{2}-s p^{2}$ & $57.3^{a}$ & 55.73 & 53.34 & 50.56 & 52.73 \\
\hline 33 & 1,2 & $s p^{2}-s p^{2}$ & $70.6^{a}$ & 72.53 & 69.36 & 66.03 & 68.92 \\
\hline 34 & 1,2 & $s p^{2}-s p^{2}$ & $69.1^{a}$ & 70.43 & 67.39 & 64.47 & 67.21 \\
\hline 35 & 1,2 & $s p^{2}-s p^{2}$ & $65.6^{a}$ & 67.77 & 65.32 & 62.09 & 64.69 \\
\hline 36 & 1,2 & $s p^{2}-s p^{2}$ & $48.54^{a}$ & 46.48 & 43.94 & 42.41 & 44.26 \\
\hline 36 & 2,3 & $s p^{2}-s p^{2}$ & $67.4^{a}$ & 70.02 & 66.82 & 63.79 & 66.52 \\
\hline 37 & 1,2 & $s p^{2}-s p^{2}$ & $58.29^{a}$ & 49.01 & 46.53 & 44.76 & 46.74 \\
\hline 38 & 1,2 & $s p^{2}-s p^{2}$ & $70.92^{a}$ & 68.83 & 66.47 & 63.41 & 66.03 \\
\hline 38 & 2,3 & $s p^{2}-s p^{2}$ & $56.63^{a}$ & 55.88 & 53.39 & 50.71 & 52.89 \\
\hline 38 & 3,4 & $s p^{2}-s p^{2}$ & $56.30^{a}$ & 55.18 & 52.54 & 49.94 & 52.09 \\
\hline 39 & 1,2 & $s p^{2}-s p^{2}$ & $61.3^{a}$ & 60.79 & 58.36 & 55.54 & 57.84 \\
\hline 39 & 2,3 & $s p^{2}-s p^{2}$ & $58.1^{a}$ & 59.13 & 56.77 & 53.71 & 55.98 \\
\hline 39 & 3,4 & $s p^{2}-s p^{2}$ & $56.2^{a}$ & 55.36 & 52.81 & 50.07 & 52.20 \\
\hline 40 & 1,2 & $s p^{2}-s p^{2}$ & $60.05^{a}$ & 57.82 & 55.71 & 52.84 & 55.08 \\
\hline 40 & 2,3 & $s p^{2}-s p^{2}$ & $56.47^{a}$ & 55.47 & 52.88 & 50.18 & 52.36 \\
\hline 40 & 3,4 & $s p^{2}-s p^{2}$ & $55.15^{a}$ & 53.97 & 51.35 & 48.75 & 50.86 \\
\hline 41 & 1,2 & $s p^{2}-s p^{2}$ & $52.78^{a}$ & 50.09 & 47.41 & 45.76 & 47.83 \\
\hline 42 & 1,2 & $s p^{2}-s p^{2}$ & $50.39^{a}$ & 47.98 & 44.83 & 43.51 & 45.47 \\
\hline 43 & 1,2 & $s p^{2}-s p^{2}$ & $57.68^{a}$ & 57.52 & 54.82 & 52.08 & 54.35 \\
\hline 43 & 1,6 & $s p^{2}-s p^{2}$ & $59.4^{a}$ & 58.77 & 57.00 & 53.93 & 56.16 \\
\hline 43 & 2,3 & $s p^{2}-s p^{2}$ & $54.71^{a}$ & 53.32 & 50.78 & 48.36 & 50.47 \\
\hline 43 & 3,4 & $s p^{2}-s p^{2}$ & $57.75^{a}$ & 57.43 & 54.90 & 52.19 & 54.47 \\
\hline 43 & 4,5 & $s p^{2}-s p^{2}$ & $70.13^{a}$ & 68.43 & 66.87 & 63.40 & 66.03 \\
\hline 43 & 6,7 & $s p^{2}-s p^{2}$ & $52.2^{a}$ & 49.96 & 48.26 & 46.06 & 47.85 \\
\hline 44 & 1,2 & $s p^{2}-s p^{2}$ & $57.1^{g}$ & 62.08 & 57.33 & 55.91 & 58.41 \\
\hline 45 & 1,2 & $s p^{2}-s p^{2}$ & $56.2^{h}$ & 54.62 & 51.99 & 49.35 & 51.49 \\
\hline 40 & 1,5 & $s p^{2}-s p$ & $80.40^{a}$ & 79.84 & 78.52 & 74.21 & 76.62 \\
\hline 46 & 1,2 & $s p^{2}-s p$ & $91.1^{a}$ & 90.64 & 90.43 & 85.59 & 88.51 \\
\hline 47 & & $s p-s p$ & $170.6^{a}$ & 201.13 & 194.42 & 186.42 & 191.26 \\
\hline 48 & 1,2 & $s p-s p$ & $175.9^{a}$ & 201.19 & 195.51 & 187.21 & 192.41 \\
\hline 49 & 1,2 & $s p-s p$ & $155.8^{a}$ & 159.56 & 158.75 & 149.28 & 154.32 \\
\hline 50 & 1,3 & $s p^{3}-s p^{3}$ & $-17.49^{i}$ & -19.08 & -22.11 & -19.83 & -20.46 \\
\hline 35 & $\operatorname{tau} .^{j}$ & $s p^{2}-s p^{2}$ & $58.29^{a}$ & 58.59 & 56.03 & 53.43 & 55.74 \\
\hline 51 & 1,2 & $s p^{2}-s p^{2}$ & $59.10^{k}$ & 59.38 & 54.95 & 53.56 & 55.97 \\
\hline
\end{tabular}

(See footnote in Table 1) 
Table 3: One-bond $\mathrm{C}-\mathrm{C}$ coupling constants (in $\mathrm{Hz}$ ). Experimental and values calculated using B3LYP functional and 6-311G**, TZVP, EPR-III and aug-cc-pVTZ-J basis set.

\begin{tabular}{|c|c|c|c|c|c|c|c|}
\hline \multirow[t]{2}{*}{ Molec. } & \multirow[t]{2}{*}{ Coupling } & \multirow[t]{2}{*}{ Type } & \multirow[t]{2}{*}{${ }^{1} J_{C C}^{e x p}$} & \multicolumn{4}{|c|}{${ }^{1} J_{C C}^{c a l}$ B3LYP functional } \\
\hline & & & & $6-311 \mathrm{G}^{* *}$ & TZVP & EPR-III & aug-cc-pVTZ-J \\
\hline 1 & & $s p^{3}-s p^{3}$ & $34.6^{a}$ & 35.16 & 34.39 & 33.42 & 34.45 \\
\hline 2 & & $s p^{3}-s p^{3}$ & $38.2^{a}$ & 39.28 & 38.10 & 36.93 & 38.18 \\
\hline 3 & 1,2 & $s p^{3}-s p^{3}$ & $33.0^{a}$ & 33.96 & 33.48 & 32.59 & 33.60 \\
\hline 4 & & $s p^{3}-s p^{3}$ & $12.4^{b}$ & 13.99 & 11.91 & 11.89 & 12.42 \\
\hline 5 & & $s p^{3}-s p^{3}$ & $28.0^{c}$ & 29.11 & 27.03 & 26.26 & 27.33 \\
\hline 6 & & $s p^{3}-s p^{3}$ & $21.0^{c}$ & 22.90 & 20.75 & 20.29 & 21.13 \\
\hline 7 & 1,2 & $s p^{3}-s p^{3}$ & $10.9^{a}$ & 11.96 & 10.55 & 10.72 & 11.18 \\
\hline 8 & & $s p^{3}-s p^{3}$ & $28.4^{b}$ & 29.00 & 27.69 & 27.55 & 28.33 \\
\hline 9 & & $s p^{3}-s p^{3}$ & $29.54^{a}$ & 29.32 & 28.19 & 28.23 & 29.04 \\
\hline 10 & 1,2 & $s p^{3}-s p^{3}$ & $21.0^{a}$ & 22.23 & 21.86 & 21.26 & 22.13 \\
\hline 10 & 1,3 & $s p^{3}-s p^{3}$ & $-17.49^{a}$ & -14.51 & -16.84 & -15.01 & -15.29 \\
\hline 11 & 1,2 & $s p^{3}-s p^{3}$ & $22^{a}$ & 23.38 & 23.17 & 22.52 & 23.40 \\
\hline 12 & 1,2 & $s p^{3}-s p^{3}$ & $36.7^{a}$ & 36.54 & 36.75 & 35.94 & 37.04 \\
\hline 12 & 2,3 & $s p^{3}-s p^{3}$ & $16.0^{a}$ & 16.35 & 15.71 & 15.50 & 16.15 \\
\hline 13 & 1,2 & $s p^{3}-s p^{3}$ & $20.2^{d}$ & 22.65 & 20.77 & 20.19 & 21.04 \\
\hline 14 & 1,2 & $s p^{3}-s p^{3}$ & $9.9^{d}$ & 8.96 & 8.27 & 8.82 & 9.35 \\
\hline 15 & & $s p^{3}-s p^{3}$ & $32.6^{b}$ & 33.03 & 32.64 & 31.79 & 32.76 \\
\hline 16 & & $s p^{3}-s p^{3}$ & $29.4^{e}$ & 27.32 & 27.83 & 28.18 & 28.88 \\
\hline 17 & 1,2 & $s p^{3}-s p^{3}$ & $25.3^{f}$ & 23.00 & 23.86 & 23.90 & 24.60 \\
\hline 18 & 1,2 & $s p^{3}-s p^{2}$ & $41.9^{a}$ & 43.83 & 43.49 & 41.66 & 43.08 \\
\hline 19 & 1,2 & $s p^{3}-s p^{2}$ & $44.9^{a}$ & 46.04 & 46.03 & 44.07 & 45.55 \\
\hline 20 & 1,3 & $s p^{3}-s p^{2}$ & $44.19^{a}$ & 45.74 & 45.99 & 43.90 & 45.30 \\
\hline 21 & & $s p^{3}-s p^{2}$ & $40.51^{a}$ & 42.12 & 40.91 & 39.33 & 40.67 \\
\hline 22 & & $s p^{3}-s p^{2}$ & $48.48^{a}$ & 53.49 & 53.22 & 50.56 & 52.31 \\
\hline 23 & 1,2 & $s p^{3}-s p^{2}$ & $49.3^{a}$ & 53.08 & 53.09 & 50.46 & 52.22 \\
\hline 23 & 1,3 & $s p^{3}-s p^{2}$ & $41.4^{a}$ & 42.30 & 41.35 & 39.76 & 41.12 \\
\hline 24 & 1,2 & $s p^{3}-s p^{2}$ & $45.91^{a}$ & 47.79 & 48.23 & 46.01 & 47.46 \\
\hline 25 & & $s p^{3}-s p^{2}$ & $40.44^{a}$ & 41.24 & 40.47 & 39.00 & 40.42 \\
\hline 26 & & $s p^{3}-s p^{2}$ & $39.4^{a}$ & 41.37 & 39.84 & 38.29 & 39.70 \\
\hline 27 & & $s p^{3}-s p^{2}$ & $56.7^{a}$ & 61.02 & 60.70 & 57.96 & 59.93 \\
\hline 3 & 2,3 & $s p^{3}-s p$ & $55.24^{a}$ & 65.75 & 63.28 & 59.97 & 61.93 \\
\hline 7 & 2,3 & $s p^{3}-s p$ & $77.9^{a}$ & 90.77 & 90.09 & 84.72 & 87.46 \\
\hline 28 & & $s p^{3}-s p$ & $56.48^{a}$ & 67.72 & 65.65 & 62.14 & 64.17 \\
\hline 29 & 1,2 & $s p^{3}-s p$ & $54.8^{a}$ & 64.28 & 61.98 & 58.75 & 60.68 \\
\hline 30 & 1,2 & $s p^{3}-s p$ & $52.0^{a}$ & 63.24 & 61.33 & 58.15 & 60.04 \\
\hline 31 & 1,2 & $s p^{3}-s p$ & $67.4^{a}$ & 76.05 & 75.28 & 71.21 & 73.50 \\
\hline
\end{tabular}


Deviations form the experimental ${ }^{1} J_{C C}^{e x p}$ of the scaled coupling constants, continued...

\begin{tabular}{|c|c|c|c|c|c|c|c|}
\hline \multirow[t]{2}{*}{ Molec. } & \multirow[t]{2}{*}{ Coupling } & \multirow[t]{2}{*}{ Type } & \multirow[t]{2}{*}{${ }^{1} J_{C C}^{e x p}$} & \multicolumn{4}{|c|}{${ }^{1} J_{C C}^{c a l}$ B3LYP functional } \\
\hline & & & & $6-311 \mathrm{G}^{* *}$ & TZVP & EPR-III & aug-cc-pVTZ-J \\
\hline 32 & & $s p^{2}-s p^{2}$ & $67.6^{a}$ & 80.19 & 77.02 & 72.36 & 75.43 \\
\hline 18 & 2,3 & $s p^{2}-s p^{2}$ & $70.0^{a}$ & 81.93 & 79.40 & 74.60 & 77.69 \\
\hline 19 & 2,3 & $s p^{2}-s p^{2}$ & $73.8^{a}$ & 85.41 & 83.45 & 78.40 & 81.60 \\
\hline 20 & 1,2 & $s p^{2}-s p^{2}$ & $57.3^{a}$ & 64.25 & 62.80 & 58.74 & 61.16 \\
\hline 33 & 1,2 & $s p^{2}-s p^{2}$ & $70.6^{a}$ & 83.40 & 81.33 & 76.49 & 79.66 \\
\hline 34 & 1,2 & $s p^{2}-s p^{2}$ & $69.1^{a}$ & 80.06 & 77.97 & 73.70 & 76.69 \\
\hline 35 & 1,2 & $s p^{2}-s p^{2}$ & $65.6^{a}$ & 76.55 & 75.01 & 70.45 & 73.28 \\
\hline 36 & 1,2 & $s p^{2}-s p^{2}$ & $48.54^{a}$ & 55.00 & 53.37 & 50.64 & 52.75 \\
\hline 36 & 2,3 & $s p^{2}-s p^{2}$ & $67.4^{a}$ & 78.51 & 76.20 & 71.96 & 74.93 \\
\hline 37 & 1,2 & $s p^{2}-s p^{2}$ & $58.29^{a}$ & 57.98 & 56.42 & 53.34 & 55.59 \\
\hline 38 & 1,2 & $s p^{2}-s p^{2}$ & $70.92^{a}$ & 78.52 & 77.24 & 72.80 & 75.70 \\
\hline 38 & 2,3 & $s p^{2}-s p^{2}$ & $56.63^{a}$ & 64.67 & 63.15 & 59.17 & 61.61 \\
\hline 38 & 3,4 & $s p^{2}-s p^{2}$ & $56.30^{a}$ & 63.85 & 62.17 & 58.29 & 60.71 \\
\hline 39 & 1,2 & $s p^{2}-s p^{2}$ & $61.3^{a}$ & 69.32 & 67.83 & 63.79 & 66.34 \\
\hline 39 & 2,3 & $s p^{2}-s p^{2}$ & $58.1^{a}$ & 67.76 & 66.36 & 62.02 & 64.53 \\
\hline 39 & 3,4 & $s p^{2}-s p^{2}$ & $56.2^{a}$ & 63.97 & 62.37 & 58.36 & 60.76 \\
\hline 40 & 1,2 & $s p^{2}-s p^{2}$ & $60.05^{a}$ & 66.71 & 65.63 & 61.45 & 63.96 \\
\hline 40 & 2,3 & $s p^{2}-s p^{2}$ & $56.47^{a}$ & 64.23 & 62.64 & 58.63 & 61.07 \\
\hline 40 & 3,4 & $s p^{2}-s p^{2}$ & $55.15^{a}$ & 62.70 & 61.05 & 57.17 & 59.56 \\
\hline 41 & 1,2 & $s p^{2}-s p^{2}$ & $52.78^{a}$ & 58.96 & 57.23 & 54.33 & 56.70 \\
\hline 42 & 1,2 & $s p^{2}-s p^{2}$ & $50.39^{a}$ & 57.25 & 55.05 & 52.45 & 54.72 \\
\hline 43 & 1,2 & $s p^{2}-s p^{2}$ & $57.68^{a}$ & 66.26 & 64.54 & 60.49 & 63.00 \\
\hline 43 & 1,6 & $s p^{2}-s p^{2}$ & $59.4^{a}$ & 67.35 & 66.59 & 62.18 & 64.63 \\
\hline 43 & 2,3 & $s p^{2}-s p^{2}$ & $54.71^{a}$ & 61.92 & 60.36 & 56.64 & 58.99 \\
\hline 43 & 3,4 & $s p^{2}-s p^{2}$ & $57.75^{a}$ & 66.12 & 64.57 & 60.55 & 63.06 \\
\hline 43 & 4,5 & $s p^{2}-s p^{2}$ & $70.13^{a}$ & 77.55 & 77.05 & 72.19 & 75.05 \\
\hline 43 & 6,7 & $s p^{2}-s p^{2}$ & $52.2^{a}$ & 58.02 & 57.25 & 53.86 & 55.86 \\
\hline 44 & 1,2 & $s p^{2}-s p^{2}$ & $57.1^{g}$ & 72.35 & 68.26 & 65.45 & 68.13 \\
\hline 45 & 1,2 & $s p^{2}-s p^{2}$ & $56.2^{h}$ & 63.29 & 61.60 & 57.64 & 60.03 \\
\hline 40 & 1,5 & $s p^{2}-s p$ & $80.40^{a}$ & 92.75 & 92.70 & 86.58 & 89.34 \\
\hline 46 & 1,2 & $s p^{2}-s p$ & $91.1^{a}$ & 102.22 & 103.35 & 96.75 & 99.93 \\
\hline 47 & & $s p-s p$ & $170.6^{a}$ & 217.06 & 210.32 & 200.63 & 205.45 \\
\hline 48 & 1,2 & $s p-s p$ & $175.9^{a}$ & 217.50 & 212.02 & 201.85 & 207.00 \\
\hline 49 & 1,2 & $s p-s p$ & $155.8^{a}$ & 179.34 & 180.05 & 168.09 & 173.50 \\
\hline 50 & 1,3 & $s p^{3}-s p^{3}$ & $-17.49^{i}$ & -18.78 & -21.76 & -19.40 & -19.93 \\
\hline 35 & $\operatorname{tau} .^{j}$ & $s p^{2}-s p^{2}$ & $58.29^{a}$ & 66.91 & 65.24 & 61.40 & 63.94 \\
\hline 51 & 1,2 & $s p^{2}-s p^{2}$ & $59.10^{k}$ & 69.29 & 65.55 & 62.81 & 65.39 \\
\hline
\end{tabular}

(See footnote in Table 1) 
Table 4: One-bond $\mathrm{C}-\mathrm{C}$ coupling constants (in $\mathrm{Hz}$ ). Experimental and values calculated using B3P86 functional and 6-311G**, TZVP, EPR-III and aug-cc-pVTZ-J basis set.

\begin{tabular}{|c|c|c|c|c|c|c|c|}
\hline \multirow[t]{2}{*}{ Molec. } & \multirow[t]{2}{*}{ Coupling } & \multirow[t]{2}{*}{ Type } & \multirow[t]{2}{*}{${ }^{1} J_{C C}^{e x p}$} & \multicolumn{4}{|c|}{${ }^{1} J_{C C}^{c a l}$ B3P86 functional } \\
\hline & & & & $6-311 \mathrm{G}^{* *}$ & TZVP & EPR-III & aug-cc-pVTZ-J \\
\hline 1 & & $s p^{3}-s p^{3}$ & $34.6^{a}$ & 30.43 & 29.42 & 29.38 & 30.35 \\
\hline 2 & & $s p^{3}-s p^{3}$ & $38.2^{a}$ & 34.45 & 32.97 & 32.84 & 33.99 \\
\hline 3 & 1,2 & $s p^{3}-s p^{3}$ & $33.0^{a}$ & 29.36 & 28.67 & 28.70 & 29.66 \\
\hline 4 & & $s p^{3}-s p^{3}$ & $12.4^{b}$ & 11.08 & 8.97 & 9.59 & 10.07 \\
\hline 5 & & $s p^{3}-s p^{3}$ & $28.0^{c}$ & 25.41 & 23.15 & 23.18 & 24.17 \\
\hline 6 & & $s p^{3}-s p^{3}$ & $21.0^{c}$ & 19.56 & 17.30 & 17.57 & 18.34 \\
\hline 7 & 1,2 & $s p^{3}-s p^{3}$ & $10.9^{a}$ & 9.24 & 7.80 & 8.59 & 9.01 \\
\hline 8 & & $s p^{3}-s p^{3}$ & $28.4^{b}$ & 24.64 & 23.23 & 24.05 & 24.77 \\
\hline 9 & & $s p^{3}-s p^{3}$ & $29.54^{a}$ & 24.94 & 23.65 & 24.67 & 25.42 \\
\hline 10 & 1,2 & $s p^{3}-s p^{3}$ & $21.0^{a}$ & 18.61 & 18.14 & 18.36 & 19.17 \\
\hline 10 & 1,3 & $s p^{3}-s p^{3}$ & $-17.49^{a}$ & -14.98 & -17.22 & -15.22 & -15.51 \\
\hline 11 & 1,2 & $s p^{3}-s p^{3}$ & $22^{a}$ & 19.95 & 19.65 & 19.80 & 20.62 \\
\hline 12 & 1,2 & $s p^{3}-s p^{3}$ & $36.7^{a}$ & 31.76 & 31.80 & 32.06 & 33.08 \\
\hline 12 & 2,3 & $s p^{3}-s p^{3}$ & $16.0^{a}$ & 13.36 & 12.68 & 13.16 & 13.76 \\
\hline 13 & 1,2 & $s p^{3}-s p^{3}$ & $20.2^{d}$ & 19.36 & 17.39 & 17.61 & 18.39 \\
\hline 14 & 1,2 & $s p^{3}-s p^{3}$ & $9.9^{d}$ & 5.74 & 4.94 & 6.32 & 6.81 \\
\hline 15 & & $s p^{3}-s p^{3}$ & $32.6^{b}$ & 28.81 & 28.28 & 28.34 & 29.24 \\
\hline 16 & & $s p^{3}-s p^{3}$ & $29.4^{e}$ & 22.96 & 23.45 & 24.85 & 25.50 \\
\hline 17 & 1,2 & $s p^{3}-s p^{3}$ & $25.3^{f}$ & 19.11 & 19.88 & 20.81 & 21.47 \\
\hline 18 & 1,2 & $s p^{3}-s p^{2}$ & $41.9^{a}$ & 38.74 & 38.11 & 37.42 & 38.74 \\
\hline 19 & 1,2 & $s p^{3}-s p^{2}$ & $44.9^{a}$ & 40.97 & 40.63 & 39.80 & 41.19 \\
\hline 20 & 1,3 & $s p^{3}-s p^{2}$ & $44.19^{a}$ & 40.83 & 40.73 & 39.75 & 41.05 \\
\hline 21 & & $s p^{3}-s p^{2}$ & $40.51^{a}$ & 37.04 & 35.55 & 35.08 & 36.32 \\
\hline 22 & & $s p^{3}-s p^{2}$ & $48.48^{a}$ & 48.05 & 47.41 & 45.97 & 47.61 \\
\hline 23 & 1,2 & $s p^{3}-s p^{2}$ & $49.3^{a}$ & 47.92 & 47.61 & 46.17 & 47.82 \\
\hline 23 & 1,3 & $s p^{3}-s p^{2}$ & $41.4^{a}$ & 37.42 & 36.23 & 35.72 & 36.99 \\
\hline 24 & 1,2 & $s p^{3}-s p^{2}$ & $45.91^{a}$ & 42.77 & 42.85 & 41.75 & 43.11 \\
\hline 25 & & $s p^{3}-s p^{2}$ & $40.44^{a}$ & 36.57 & 35.51 & 35.08 & 36.40 \\
\hline 26 & & $s p^{3}-s p^{2}$ & $39.4^{a}$ & 36.45 & 34.60 & 34.13 & 35.43 \\
\hline 27 & & $s p^{3}-s p^{2}$ & $56.7^{a}$ & 55.22 & 54.48 & 52.95 & 54.79 \\
\hline 3 & 2,3 & $s p^{3}-s p$ & $55.24^{a}$ & 59.13 & 56.33 & 54.44 & 56.29 \\
\hline 7 & 2,3 & $s p^{3}-s p$ & $77.9^{a}$ & 83.27 & 82.17 & 78.36 & 80.94 \\
\hline 28 & & $s p^{3}-s p$ & $56.48^{a}$ & 60.73 & 58.23 & 56.14 & 58.05 \\
\hline 29 & 1,2 & $s p^{3}-s p$ & $54.8^{a}$ & 57.94 & 55.41 & 53.61 & 55.42 \\
\hline 30 & 1,2 & $s p^{3}-s p$ & $52.0^{a}$ & 57.15 & 55.11 & 53.33 & 55.12 \\
\hline 31 & 1,2 & $s p^{3}-s p$ & $67.4^{a}$ & 69.09 & 67.90 & 65.31 & 67.50 \\
\hline
\end{tabular}


Deviations form the experimental ${ }^{1} J_{C C}^{e x p}$ of the scaled coupling constants, continued...

\begin{tabular}{|c|c|c|c|c|c|c|c|}
\hline \multirow[t]{2}{*}{ Molec. } & \multirow[t]{2}{*}{ Coupling } & \multirow[t]{2}{*}{ Type } & \multirow[t]{2}{*}{${ }^{1} J_{C C}^{e x p}$} & \multicolumn{4}{|c|}{${ }^{1} J_{C C}^{c a l}$ B3P86 functional } \\
\hline & & & & $6-311 \mathrm{G}^{* *}$ & TZVP & EPR-III & aug-cc-pVTZ-J \\
\hline 32 & & $s p^{2}-s p^{2}$ & $67.6^{a}$ & 73.24 & 69.81 & 66.85 & 69.67 \\
\hline 18 & 2,3 & $s p^{2}-s p^{2}$ & $70.0^{a}$ & 75.27 & 72.47 & 69.37 & 72.23 \\
\hline 19 & 2,3 & $s p^{2}-s p^{2}$ & $73.8^{a}$ & 78.82 & 76.52 & 73.21 & 76.19 \\
\hline 20 & 1,2 & $s p^{2}-s p^{2}$ & $57.3^{a}$ & 58.95 & 57.18 & 54.55 & 56.82 \\
\hline 33 & 1,2 & $s p^{2}-s p^{2}$ & $70.6^{a}$ & 76.56 & 74.13 & 71.02 & 73.96 \\
\hline 34 & 1,2 & $s p^{2}-s p^{2}$ & $69.1^{a}$ & 73.78 & 71.35 & 68.63 & 71.40 \\
\hline 35 & 1,2 & $s p^{2}-s p^{2}$ & $65.6^{a}$ & 70.47 & 68.62 & 65.55 & 68.18 \\
\hline 36 & 1,2 & $s p^{2}-s p^{2}$ & $48.54^{a}$ & 49.85 & 47.89 & 46.40 & 48.34 \\
\hline 36 & 2,3 & $s p^{2}-s p^{2}$ & $67.4^{a}$ & 72.48 & 69.88 & 67.12 & 69.87 \\
\hline 37 & 1,2 & $s p^{2}-s p^{2}$ & $58.29^{a}$ & 52.62 & 50.71 & 48.92 & 50.98 \\
\hline 38 & 1,2 & $s p^{2}-s p^{2}$ & $70.92^{a}$ & 72.67 & 70.99 & 68.07 & 70.78 \\
\hline 38 & 2,3 & $s p^{2}-s p^{2}$ & $56.63^{a}$ & 59.13 & 57.25 & 54.68 & 56.95 \\
\hline 38 & 3,4 & $s p^{2}-s p^{2}$ & $56.30^{a}$ & 58.29 & 56.30 & 53.84 & 56.10 \\
\hline 39 & 1,2 & $s p^{2}-s p^{2}$ & $61.3^{a}$ & 63.90 & 62.08 & 59.42 & 61.81 \\
\hline 39 & 2,3 & $s p^{2}-s p^{2}$ & $58.1^{a}$ & 62.11 & 60.34 & 57.46 & 59.79 \\
\hline 39 & 3,4 & $s p^{2}-s p^{2}$ & $56.2^{a}$ & 58.44 & 56.51 & 53.93 & 56.16 \\
\hline 40 & 1,2 & $s p^{2}-s p^{2}$ & $60.05^{a}$ & 61.34 & 59.89 & 57.13 & 59.48 \\
\hline 40 & 2,3 & $s p^{2}-s p^{2}$ & $56.47^{a}$ & 58.74 & 56.77 & 54.21 & 56.47 \\
\hline 40 & 3,4 & $s p^{2}-s p^{2}$ & $55.15^{a}$ & 57.20 & 55.22 & 52.76 & 54.98 \\
\hline 41 & 1,2 & $s p^{2}-s p^{2}$ & $52.78^{a}$ & 53.61 & 51.56 & 50.01 & 52.20 \\
\hline 42 & 1,2 & $s p^{2}-s p^{2}$ & $50.39^{a}$ & 51.99 & 49.44 & 48.17 & 50.26 \\
\hline 43 & 1,2 & $s p^{2}-s p^{2}$ & $57.68^{a}$ & 60.65 & 58.56 & 55.96 & 58.30 \\
\hline 43 & 1,6 & $s p^{2}-s p^{2}$ & $59.4^{a}$ & 62.01 & 60.85 & 57.82 & 60.12 \\
\hline 43 & 2,3 & $s p^{2}-s p^{2}$ & $54.71^{a}$ & 56.44 & 54.54 & 52.23 & 54.42 \\
\hline 43 & 3,4 & $s p^{2}-s p^{2}$ & $57.75^{a}$ & 60.56 & 58.64 & 56.05 & 58.39 \\
\hline 43 & 4,5 & $s p^{2}-s p^{2}$ & $70.13^{a}$ & 71.83 & 70.90 & 67.53 & 70.21 \\
\hline 43 & 6,7 & $s p^{2}-s p^{2}$ & $52.2^{a}$ & 53.05 & 51.91 & 49.79 & 51.65 \\
\hline 44 & 1,2 & $s p^{2}-s p^{2}$ & $57.1^{g}$ & 66.25 & 61.99 & 60.69 & 63.17 \\
\hline 45 & 1,2 & $s p^{2}-s p^{2}$ & $56.2^{h}$ & 57.76 & 55.75 & 53.22 & 55.45 \\
\hline 40 & 1,5 & $s p^{2}-s p$ & $80.40^{a}$ & 85.56 & 84.89 & 80.40 & 82.99 \\
\hline 46 & 1,2 & $s p^{2}-s p$ & $91.1^{a}$ & 95.40 & 95.93 & 90.96 & 93.99 \\
\hline 47 & & $s p-s p$ & $170.6^{a}$ & 205.42 & 199.02 & 191.68 & 196.14 \\
\hline 48 & 1,2 & $s p-s p$ & $175.9^{a}$ & 206.86 & 201.44 & 193.47 & 198.28 \\
\hline 49 & 1,2 & $s p-s p$ & $155.8^{a}$ & 168.50 & 168.24 & 158.47 & 163.67 \\
\hline 50 & 1,3 & $s p^{3}-s p^{3}$ & $-17.49^{i}$ & -18.95 & -21.73 & -19.39 & -19.92 \\
\hline 35 & $\operatorname{tau} .^{j}$ & $s p^{2}-s p^{2}$ & $58.29^{a}$ & 61.30 & 59.32 & 56.84 & 59.18 \\
\hline 51 & 1,2 & $s p^{2}-s p^{2}$ & $59.10^{k}$ & 63.54 & 59.63 & 58.43 & 60.83 \\
\hline
\end{tabular}

(See footnote in Table 1) 
Table 5: Deviations from the experimental ${ }^{1} J_{C C}^{e x p}$ of the scaled ${ }^{a}$

${ }^{1} J_{C C}^{\text {scaled }}$ coupling constants.

\begin{tabular}{|c|c|c|c|c|c|c|c|c|c|c|c|c|c|c|c|c|c|c|c|c|}
\hline Molec. & Coup. & type & ${ }^{1} J_{C C}^{e x p}$ & (1) & (2) & (3) & (4) & (5) & (6) & (7) & (8) & (9) & (10) & (11) & (12) & (13) & (14) & (15) & (16) & Aver. \\
\hline 1 & & $s p^{3}-s p^{3}$ & 34.6 & 1.7 & 1.6 & 1.4 & 1.6 & 1.8 & 1.7 & 1.5 & 1.6 & 1.5 & 1.4 & 1.1 & 1.3 & 1.9 & 1.7 & 1.6 & 1.7 & 1.5 \\
\hline 3 & 1,2 & $s p^{3}-s p^{3}$ & 33.0 & 1.1 & 0.8 & 0.6 & 0.7 & 1.2 & 0.8 & 0.6 & 0.7 & 0.9 & 0.5 & 0.3 & 0.4 & 1.2 & 0.8 & 0.6 & 0.8 & 0.7 \\
\hline 5 & & $s p^{3}-s p^{3}$ & 28.0 & -1.1 & -0.1 & 0.0 & -0.1 & -1.4 & -0.4 & -0.2 & -0.3 & -0.1 & 0.9 & 1.0 & 0.9 & -0.4 & 0.6 & 0.8 & 0.7 & 0.0 \\
\hline 6 & & $s p^{3}-s p^{3}$ & 21.0 & -2.9 & -1.9 & -1.6 & -1.7 & -3.2 & -2.1 & -1.7 & -1.8 & -2.0 & -0.9 & -0.7 & -0.8 & -2.4 & -1.3 & -0.9 & -1.0 & -1.6 \\
\hline 7 & 1,2 & $s p^{3}-s p^{3}$ & 10.9 & -3.8 & -3.4 & -3.0 & -3.1 & -4.1 & -3.6 & -3.0 & -3.1 & -3.2 & -2.6 & -2.2 & -2.3 & -3.6 & -3.1 & -2.6 & -2.7 & -2.9 \\
\hline 8 & & $s p^{3}-s p^{3}$ & 28.4 & 0.8 & 1.3 & 0.8 & 1.0 & 1.1 & 1.5 & 0.9 & 1.1 & 0.4 & 0.7 & 0.2 & 0.4 & 0.6 & 0.9 & 0.4 & 0.6 & 0.8 \\
\hline 10 & 1,2 & $s p^{3}-s p^{3}$ & 21.0 & -1.7 & -2.1 & -1.8 & -1.9 & -1.8 & -2.2 & -1.9 & -2.0 & -1.5 & -1.9 & -1.5 & -1.6 & -1.6 & -2.0 & -1.7 & -1.8 & -1.7 \\
\hline 10 & 1,3 & $s p^{3}-s p^{3}$ & -17.5 & -11.8 & -10.5 & -9.7 & -10.0 & -12.4 & -11.0 & -9.7 & -10.0 & -9.9 & -8.3 & -7.4 & -7.7 & -11.2 & -9.6 & -8.6 & -8.9 & -9.2 \\
\hline 11 & 1,2 & $s p^{3}-s p^{3}$ & 22.0 & -1.5 & -2.1 & -1.9 & -1.9 & -1.7 & -2.3 & -1.9 & -2.0 & -1.4 & -2.0 & -1.7 & -1.7 & -1.7 & -2.3 & -2.0 & -2.1 & -1.8 \\
\hline 12 & 1,2 & $s p^{3}-s p^{3}$ & 36.7 & 3.2 & 2.2 & 1.7 & 1.9 & 3.4 & 2.4 & 1.8 & 1.9 & 2.5 & 1.5 & 1.0 & 1.2 & 2.8 & 1.8 & 1.2 & 1.4 & 1.9 \\
\hline 12 & 2,3 & $s p^{3}-s p^{3}$ & 16.0 & -2.3 & -2.5 & -2.1 & -2.2 & -2.5 & -2.7 & -2.2 & -2.3 & -1.7 & -1.8 & -1.4 & -1.5 & -2.1 & -2.3 & -1.8 & -1.9 & -1.9 \\
\hline 13 & 1,2 & $s p^{3}-s p^{3}$ & 20.2 & -3.0 & -2.1 & -1.7 & -1.8 & -3.0 & -2.2 & -1.7 & -1.8 & -2.6 & -1.8 & -1.4 & -1.5 & -3.0 & -2.2 & -1.7 & -1.8 & -2.0 \\
\hline 14 & 1,2 & $s p^{3}-s p^{3}$ & 9.9 & -2.2 & -2.2 & -1.9 & -2.1 & -2.0 & -1.9 & -1.7 & -1.9 & -1.7 & -1.7 & -1.4 & -1.7 & -1.6 & -1.6 & -1.4 & -1.7 & -1.7 \\
\hline 19 & 1,2 & $s p^{3}-s p^{2}$ & 44.9 & 3.3 & 2.5 & 2.3 & 2.5 & 3.4 & 2.6 & 2.4 & 2.5 & 2.9 & 2.0 & 1.8 & 2.0 & 3.1 & 2.3 & 2.1 & 2.2 & 2.3 \\
\hline 20 & 1,3 & $s p^{3}-s p^{2}$ & 44.2 & 2.5 & 1.5 & 1.4 & 1.7 & 2.7 & 1.6 & 1.5 & 1.7 & 2.4 & 1.4 & 1.3 & 1.5 & 2.5 & 1.5 & 1.4 & 1.6 & 1.7 \\
\hline 21 & & $s p^{3}-s p^{2}$ & 40.5 & 2.5 & 2.8 & 2.7 & 2.8 & 2.6 & 2.9 & 2.7 & 2.8 & 1.7 & 1.9 & 1.7 & 1.8 & 2.1 & 2.3 & 2.1 & 2.2 & 2.2 \\
\hline 22 & & $s p^{3}-s p^{2}$ & 48.5 & -0.5 & -1.3 & -1.3 & -1.1 & -0.3 & -1.1 & -1.2 & -1.1 & 0.4 & -0.3 & -0.4 & -0.3 & 0.6 & -0.1 & -0.1 & 0.0 & -0.5 \\
\hline 23 & 1,2 & $s p^{3}-s p^{2}$ & 49.3 & 0.4 & -0.7 & -0.8 & -0.6 & 0.6 & -0.6 & -0.6 & -0.5 & 1.5 & 0.6 & 0.5 & 0.6 & 1.5 & 0.6 & 0.5 & 0.6 & 0.2 \\
\hline 23 & 1,3 & $s p^{3}-s p^{2}$ & 41.4 & 2.9 & 2.9 & 2.8 & 2.9 & 2.9 & 2.9 & 2.8 & 2.8 & 2.5 & 2.4 & 2.2 & 2.3 & 2.7 & 2.6 & 2.4 & 2.5 & 2.5 \\
\hline 24 & 1,2 & $s p^{3}-s p^{2}$ & 45.9 & 2.6 & 1.3 & 1.2 & 1.5 & 2.7 & 1.5 & 1.3 & 1.5 & 2.5 & 1.2 & 1.1 & 1.3 & 2.6 & 1.3 & 1.3 & 1.5 & 1.6 \\
\hline
\end{tabular}


Deviations form the experimental ${ }^{1} J_{C C}^{e x p}$ of the scaled coupling constants, continued...

\begin{tabular}{|c|c|c|c|c|c|c|c|c|c|c|c|c|c|c|c|c|c|c|c|c|}
\hline Molec. & Coup. & type & ${ }^{1} J_{C C}^{e x p}$ & (1) & $(2)$ & (3) & (4) & (5) & (6) & (7) & (8) & (9) & (10) & (11) & (12) & (13) & (14) & (15) & (16) & Aver. \\
\hline 25 & & $s p^{3}-s p^{2}$ & 40.4 & 2.7 & 2.5 & 2.4 & 2.4 & 2.8 & 2.6 & 2.5 & 2.5 & 2.4 & 2.2 & 1.9 & 2.0 & 2.4 & 2.3 & 2.1 & 2.1 & 2.2 \\
\hline 26 & & $s p^{3}-s p^{2}$ & 39.4 & 2.0 & 2.6 & 2.5 & 2.5 & 2.0 & 2.6 & 2.5 & 2.5 & 1.2 & 1.7 & 1.5 & 1.6 & 1.5 & 2.0 & 1.9 & 1.9 & 1.9 \\
\hline 27 & & $s p^{3}-s p^{2}$ & 56.7 & 2.2 & 1.4 & 0.9 & 1.1 & 2.3 & 1.4 & 0.9 & 1.1 & 2.4 & 1.7 & 1.2 & 1.3 & 2.7 & 2.0 & 1.5 & 1.7 & 1.5 \\
\hline 3 & 2,3 & $s p^{3}-s p$ & 55.2 & -1.4 & -0.3 & -0.4 & -0.2 & -1.1 & -0.1 & -0.4 & -0.2 & -2.9 & -1.9 & -2.1 & -1.9 & -2.2 & -1.1 & -1.3 & -1.1 & -1.1 \\
\hline 7 & 2,3 & $s p^{3}-s p$ & 77.9 & 1.0 & 0.2 & -0.1 & 0.3 & 1.0 & 0.1 & -0.4 & 0.1 & -0.7 & -1.4 & -1.7 & -1.3 & -0.2 & -1.0 & -1.1 & -0.8 & -0.4 \\
\hline 28 & & $s p^{3}-s p$ & 56.5 & -1.6 & -0.7 & -0.9 & -0.6 & -1.4 & -0.6 & -0.9 & -0.7 & -3.3 & -2.6 & -2.8 & -2.6 & -2.3 & -1.5 & -1.7 & -1.5 & -1.5 \\
\hline 29 & 1,2 & $s p^{3}-s p$ & 54.8 & -0.8 & 0.1 & -0.1 & 0.2 & -0.6 & 0.3 & -0.1 & 0.2 & -2.1 & -1.3 & -1.5 & -1.2 & -1.6 & -0.7 & -1.0 & -0.8 & -0.6 \\
\hline 30 & 1,2 & $s p^{3}-s p$ & 52.0 & -2.9 & -2.5 & -2.6 & -2.4 & -2.7 & -2.2 & -2.6 & -2.3 & -4.1 & -3.5 & -3.7 & -3.5 & -3.7 & -3.3 & -3.5 & -3.3 & -2.9 \\
\hline 31 & 1,2 & $s p^{3}-s p$ & 67.4 & 1.0 & 0.6 & 0.1 & 0.5 & 1.3 & 0.8 & 0.2 & 0.6 & 0.8 & 0.3 & -0.1 & 0.3 & 1.4 & 1.0 & 0.6 & 0.9 & 0.6 \\
\hline 32 & & $s p^{2}-s p^{2}$ & 67.6 & -2.5 & -1.0 & -0.9 & -1.2 & -2.1 & -0.7 & -0.7 & -1.0 & -2.3 & -0.9 & -0.9 & -1.2 & -1.9 & -0.5 & -0.6 & -0.9 & -1.1 \\
\hline 18 & 2,3 & $s p^{2}-s p^{2}$ & 70.0 & 0.0 & 0.4 & -0.9 & -1.0 & -3.5 & -2.3 & -1.1 & -1.5 & -1.4 & -0.5 & -0.5 & -0.7 & -1.3 & -0.4 & -0.6 & -0.8 & -0.9 \\
\hline 19 & 2,3 & $s p^{2}-s p^{2}$ & 73.8 & -0.8 & -0.3 & -0.3 & -0.6 & -0.4 & 0.0 & -0.2 & -0.4 & -0.4 & 0.0 & -0.1 & -0.3 & -0.5 & -0.2 & -0.4 & -0.6 & -0.3 \\
\hline 20 & 1,2 & $s p^{2}-s p^{2}$ & 57.3 & -0.3 & -0.1 & 0.4 & 0.2 & -0.1 & 0.1 & 0.5 & 0.4 & 0.4 & 0.6 & 1.1 & 0.9 & 0.1 & 0.2 & 0.6 & 0.5 & 0.3 \\
\hline 33 & 1,2 & $s p^{2}-s p^{2}$ & 70.6 & -1.9 & -1.2 & -1.3 & -1.5 & -1.5 & -1.0 & -1.2 & -1.4 & -2.0 & -1.5 & -1.6 & -1.8 & -1.8 & -1.3 & -1.5 & -1.7 & -1.4 \\
\hline 34 & 1,2 & $s p^{2}-s p^{2}$ & 69.1 & -1.2 & -0.7 & -1.1 & -1.2 & -1.2 & -0.7 & -1.2 & -1.3 & -0.7 & -0.2 & -0.6 & -0.8 & -0.9 & -0.4 & -0.8 & -0.9 & -0.8 \\
\hline 35 & 1,2 & $s p^{2}-s p^{2}$ & 65.6 & -2.3 & -2.3 & -2.3 & -2.4 & -2.3 & -2.4 & -2.4 & -2.5 & -1.4 & -1.2 & -1.2 & -1.3 & -1.5 & -1.5 & -1.4 & -1.5 & -1.8 \\
\hline 36 & 1,2 & $s p^{2}-s p^{2}$ & 48.5 & -0.7 & -0.3 & -0.3 & -0.5 & -0.7 & -0.3 & -0.3 & -0.5 & -0.8 & -0.4 & -0.4 & -0.6 & -0.9 & -0.4 & -0.5 & -0.6 & -0.5 \\
\hline 36 & 2,3 & $s p^{2}-s p^{2}$ & 67.4 & -2.5 & -1.9 & -2.2 & -2.4 & -2.5 & -1.9 & -2.2 & -2.4 & -1.2 & -0.4 & -0.7 & -0.9 & -1.5 & -0.8 & -1.1 & -1.2 & -1.5 \\
\hline 37 & 1,2 & $s p^{2}-s p^{2}$ & 58.3 & 6.8 & 7.2 & 7.3 & 7.0 & 6.8 & 7.1 & 7.1 & 6.9 & 6.5 & 6.8 & 6.9 & 6.7 & 6.5 & 6.8 & 6.9 & 6.7 & 6.5 \\
\hline 38 & 1,2 & $s p^{2}-s p^{2}$ & 70.9 & 1.9 & 1.7 & 1.6 & 1.4 & 2.0 & 1.9 & 1.7 & 1.6 & 2.3 & 2.2 & 2.0 & 1.9 & 1.9 & 1.8 & 1.5 & 1.4 & 1.7 \\
\hline 38 & 2,3 & $s p^{2}-s p^{2}$ & 56.6 & -1.0 & -0.7 & -0.3 & -0.5 & -0.9 & -0.7 & -0.3 & -0.5 & -0.6 & -0.4 & 0.0 & -0.2 & -0.8 & -0.5 & -0.2 & -0.3 & -0.5 \\
\hline 38 & 3,4 & $s p^{2}-s p^{2}$ & 56.3 & -0.8 & -0.4 & 0.0 & -0.2 & -0.6 & -0.2 & 0.1 & 0.0 & -0.3 & 0.1 & 0.5 & 0.3 & -0.4 & 0.0 & 0.3 & 0.1 & -0.1 \\
\hline 39 & 1,2 & $s p^{2}-s p^{2}$ & 61.3 & -0.7 & -0.6 & -0.4 & -0.5 & -0.5 & -0.4 & -0.3 & -0.4 & 0.3 & 0.4 & 0.5 & 0.4 & -0.2 & -0.1 & 0.1 & -0.1 & -0.2 \\
\hline 39 & 2,3 & $s p^{2}-s p^{2}$ & 58.1 & -2.4 & -2.3 & -1.7 & -1.9 & -2.3 & -2.2 & -1.7 & -1.9 & -1.7 & -1.6 & -1.1 & -1.2 & -1.9 & -1.7 & -1.3 & -1.4 & -1.7 \\
\hline 39 & 3,4 & $s p^{2}-s p^{2}$ & 56.2 & -1.0 & -0.7 & -0.2 & -0.4 & -0.8 & -0.6 & -0.1 & -0.2 & -0.5 & -0.2 & 0.3 & 0.1 & -0.6 & -0.3 & 0.1 & 0.0 & -0.3 \\
\hline 40 & 1,2 & $s p^{2}-s p^{2}$ & 60.0 & 0.7 & 0.5 & 1.0 & 0.8 & 0.8 & 0.7 & 1.1 & 0.9 & 1.1 & 1.0 & 1.4 & 1.2 & 0.8 & 0.6 & 1.0 & 0.8 & 0.8 \\
\hline 40 & 2,3 & $s p^{2}-s p^{2}$ & 56.5 & -0.8 & -0.5 & 0.0 & -0.2 & -0.7 & -0.4 & 0.1 & -0.1 & -0.4 & -0.1 & 0.3 & 0.1 & -0.6 & -0.3 & 0.1 & 0.0 & -0.2 \\
\hline 40 & 3,4 & $s p^{2}-s p^{2}$ & 55.1 & -0.9 & -0.5 & 0.0 & -0.2 & -0.7 & -0.3 & 0.1 & 0.0 & -0.5 & -0.1 & 0.3 & 0.1 & -0.6 & -0.2 & 0.2 & 0.0 & -0.2 \\
\hline 41 & 1,2 & $s p^{2}-s p^{2}$ & 52.8 & 0.2 & 0.7 & 0.6 & 0.3 & 0.4 & 0.8 & 0.7 & 0.4 & 0.2 & 0.7 & 0.5 & 0.2 & 0.1 & 0.6 & 0.4 & 0.1 & 0.4 \\
\hline 42 & 1,2 & $s p^{2}-s p^{2}$ & 50.4 & -0.3 & 0.6 & 0.4 & 0.1 & -0.2 & 0.8 & 0.5 & 0.2 & -0.8 & 0.1 & -0.2 & -0.5 & -0.9 & 0.1 & -0.3 & -0.5 & 0.0 \\
\hline
\end{tabular}


Deviations form the experimental ${ }^{1} J_{C C}^{e x p}$ of the scaled coupling constants, continued...

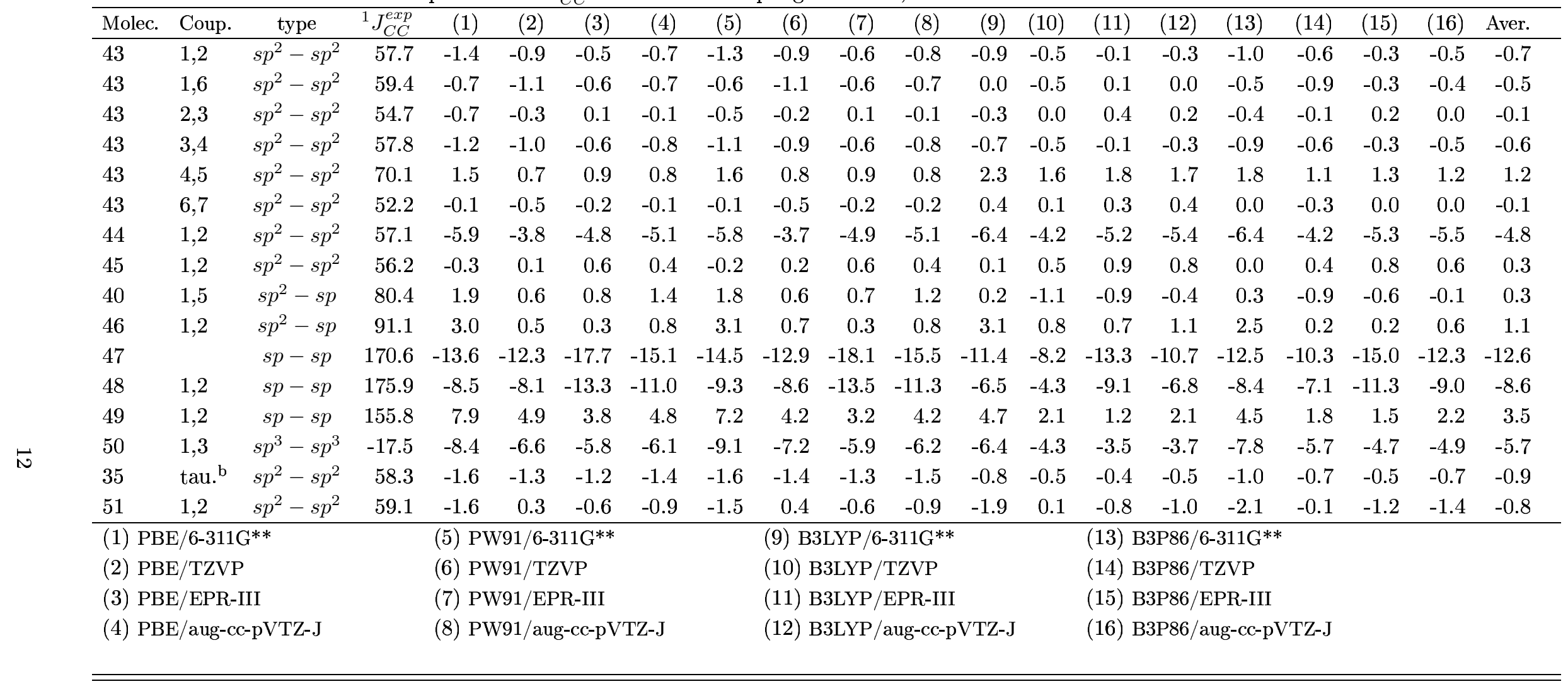

${ }^{\mathrm{a}}$ Obtained for each functional/basis set combination using the equation

$$
{ }^{1} J_{C C}^{\text {scaled }}=a+b \cdot{ }^{1} J_{C C}^{c a l c}
$$

with the coefficients $a$ and $b$ given in Table 2 of paper.

${ }^{\mathrm{b}}$ Considering a dynamic tautomerism where the experimental coupling corresponds to the average between ${ }^{1} J_{C_{1} C_{2}}$ and ${ }^{1} J_{C_{1} C_{3}}$ (see Table 5 in paper). 

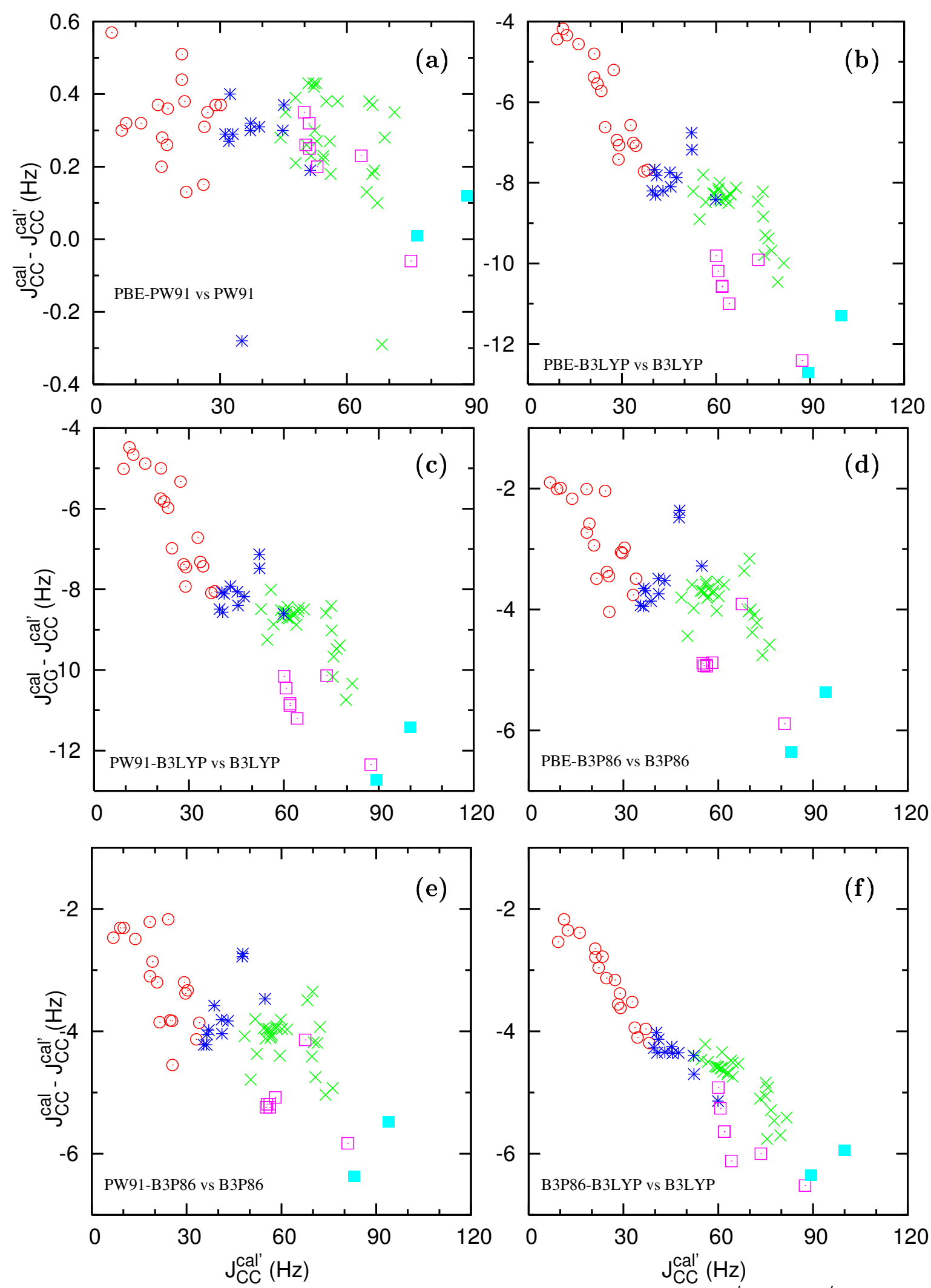

Figure 1: Differences between the results of two functional $\left({ }^{1} J_{C C}^{c a l}-{ }^{1} J_{C C}^{c a l}{ }^{\prime}\right)$ vs $\left.{ }^{1} J_{C C}^{c a l}{ }^{\prime}\right)$. aug-cc-pVTZ$\mathrm{J}$ basis set were used. ${ }^{1} J_{C_{s p} 3}-C_{s p 3}(\circ),{ }^{1} J_{C_{s p 3}-C_{s p 2}}(*),{ }^{1} J_{C_{s p 2}-C_{s p 2}}(\times),{ }^{1} J_{C_{s p 3}-C_{s p}}(\square)$, ${ }^{1} J_{C_{s p 2}-C_{s p}}$ 

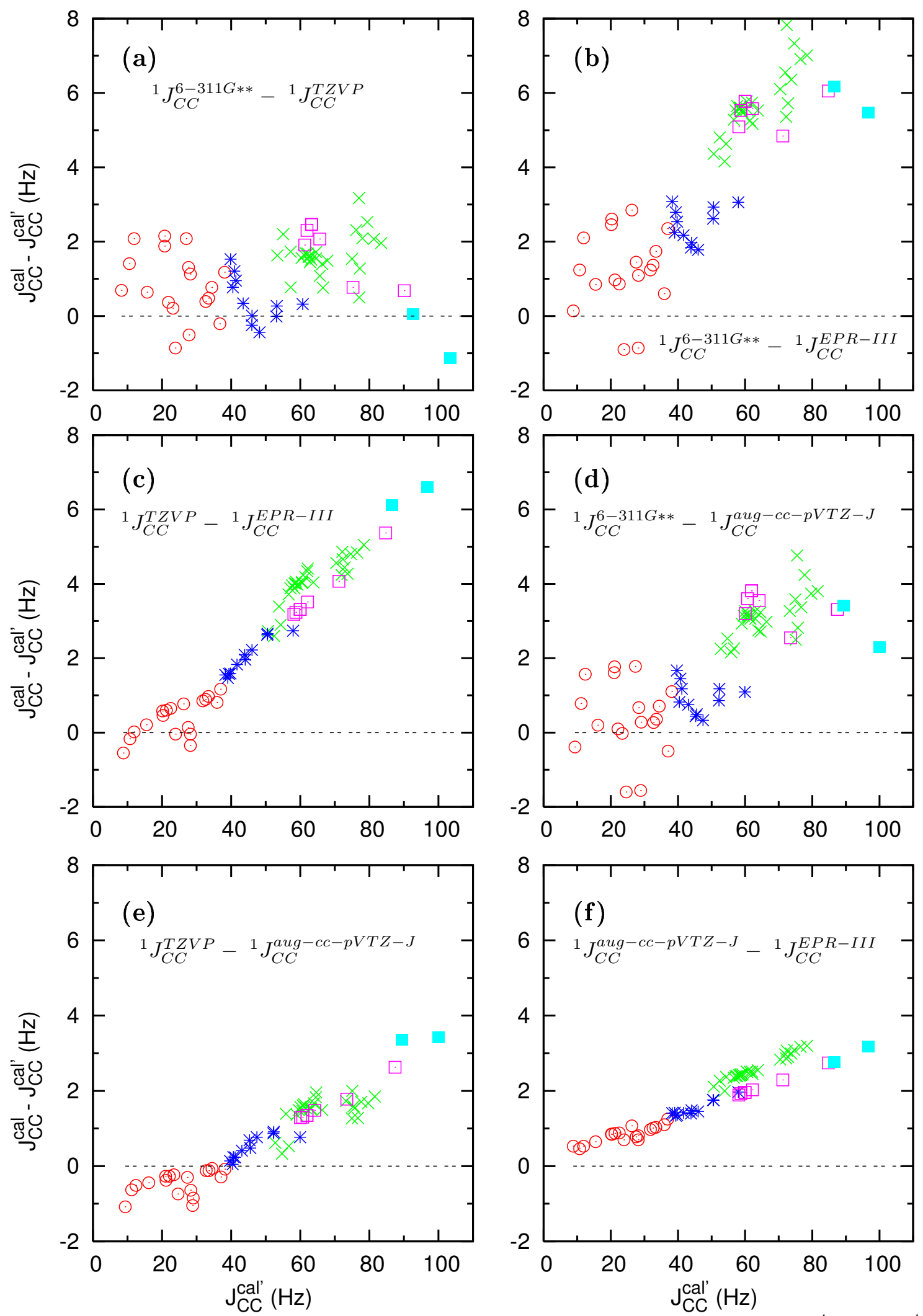

Figure 2: Differences between the results of two different basis sets $\left({ }^{1} J_{C C}^{c a l}-{ }^{1} J_{C C}^{c a l^{\prime}}\right)$ vs $\left.{ }^{1} J_{C C}^{c a l^{\prime}}\right)$. B3LYP functional were used. ${ }^{1} J_{C_{s p}{ }^{3}-C_{s p 3}}(\circ),{ }^{1} J_{C_{s p 3}-C_{s p 2}}(*),{ }^{1} J_{C_{s p 2}-C_{s p 2}}(\times),{ }^{1} J_{C_{s p 3}-C_{s p}}(\square)$, ${ }^{1} J_{C_{s p 2}-C_{s p}}$ 